\title{
Co-simulation of Stabilization Accuracy Optimization of Overhead Weapon Station
}

\author{
W. Deng, B.Q. Mao, B.W. Liang, P. Song \\ Academy of Armored Force Engineering,Beijing, China
}

Keyword: Overhead Weapon Station(OWS); Stabilization Accuracy; Multidisciplinary Collaborative Simulation; Optimization Design

\begin{abstract}
The Overhead Weapon Station(OWS) has a system composed of complex types, and its interactive subsystems make it difficult to establish the optimization model of multidisciplinary collaborative simulation. In this paper, Adams was used to establish OWS mechanical system model and Simulink to the control system model, which were integrated in multidisciplinary design optimization platform ModelCenter. What's more, taking OWS stabilization accuracy as objective function, the first order difference model was used in sensitivity analysis of control system proportion coefficient and integral coefficient. Based on design exploration tool, optimization design of the parameters was carried out. The simulation results show that, the multidisciplinary collaborative optimization model of OWS stabilization accuracy has a short design period and high calculation efficiency, and provide technical support for optimization design of OWS multi conditions and structural parameters.
\end{abstract}

\section{INTRODUCTION}

Overhead weapon station is equipped with a variety of weapons and different combinations of fire control system, can carry on the modular weapon system of different military vehicle and platform. Overhead weapon station by using multi weapon platform Launch Technology Co, high precision of biaxial stabilization technology, all digital control drive technology, high speed adjustable gun technique and a series of advanced technology, make the weapon platform capable of high precision shooting and variable firing rate under fire. At the same time, the complexity of composition of the weapons station system also caused the complexity of gun control system work process, the research difficulty increases the stability analysis of precision engineering personnel.

In this paper, ModelCenter platform for weapons simulation of electromechanical model station for system integration and optimization based on. Among them, using Adams structure overhead weapon station of rigid flexible coupling multibody dynamics model, control system model constructed by Simulink, and Simulink as the main body of the simulation environment, in the mechanical system simulation process of continuous call Adams structure model. Finally to package control system in ModelCenter, the test design function provided by ModelCenter to the weapons the United Simulation of electromechanical model station sensitivity analysis and design optimization.

\section{CONSTRUCTION OF WEAPON STATION MODEL}

A type of overhead weapon station mainly consists of a $30 \mathrm{~mm}$ chain automatic gun, the buffer spring, cradle, a supporting frame, the ear shaft, bracket, the bullet box, sighting device components, as shown in figure 1. 


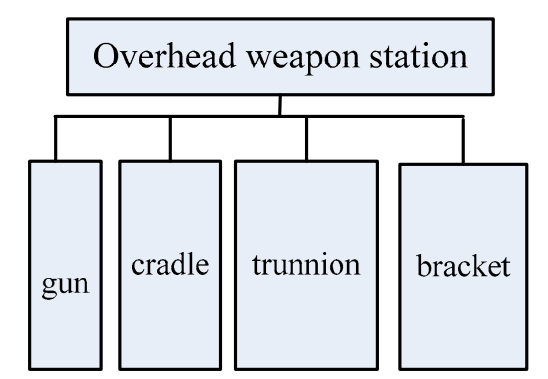

Fig 1 Composition of overhead weapon station structure

Among them, automata to perform reciprocating movement in a rocking frame and the supporting frame of the common restriction, the cradle is trunnion bearing by symmetrical with respect to the carrier for pitching, ends of the left ear shaft is fixed with the bullet box, right ear shaft is fixed with the sighting device. The level of machine is installed on the left bracket, the gear and the meshing cradle tooth arc control pitch angle of the whole part of rise and fall; bracket installed in the gun on the bed, fixed bed and the upper gun ring is fixedly connected with the chassis, the top ring, upper and lower seat through the ball to achieve relative rotation direction; installed in the gun bed last, through meshing gear and gun bed external tooth arc in control of the rotary part of the azimuth angle.

\section{CONSTRUCTION OF CONTROL SYSTEM MODEL}

A type of overhead weapon station gun control system mainly includes the servo drive mechanism, a position sensor, gyroscope, high and low speed to the power amplifier and the height to the DC motor, the horizontal power amplifier and horizontal DC motor. When the weapons station for firing on the move, as the pavement undulating form vibration excitation, tube space point shift, in steady state under the action of the gun control system, the electrical signal generated by the angular velocity gyroscope and offset proportional control system, signal processing and through the formation of phase sensitive rectifier, integrator, integrated amplifier controller, and transported to the power amplifier in pulse width modulation processing, direct drive level machine / direction of engine output torque (high and low machine acts on the cradle tooth arc gear, direction machine acting on the ring outer tooth arc), makes the body tube in the opposite direction of rotation, the eventual realization of the barrel offset angle the compensation.

In the stable condition in the process of the interaction, forming integrated control signal by using the angular velocity deviation control based on the principle of PI, as shown in figure 2:

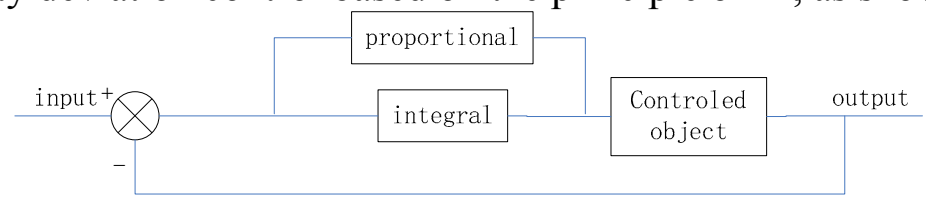

Fig. 2 schematic diagram of PI control

According to the angular velocity error signal system, using the proportional and integral two ways to calculate the amount of comprehensive control. To calculate the control quantity by the following formula derived:

$e(t)=r(t)-y(t)$

$u(t)=K_{P} e(t)+K_{I} \int e(t) d t$

According to the above analysis, the establishment of overhead weapon system of gun control system block diagram of station in Simulink, as shown in figure 3: 


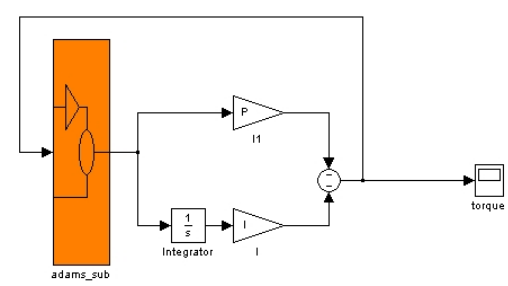

Figure 3 gun control block diagram of control system

\section{INTEGRATED SYSTEM IN MODELCENTER}

The development of Phoenix Integration, ModelCenter is a good opening, strong function of multidisciplinary collaborative design integrated optimization platform, support Adams, Matlab, Ansys and so on the many kinds of different disciplines of the CAE software. Based on the platform, the distributed modeling and optimization analysis function the user can make full use of the ModelCenter to design of complex system.

In this paper the construction of overhead weapon station joint simulation of electromechanical model, because the Adams is as a sub module is integrated into the control block diagram of the Simulink, so only in the ModelCenter control block diagram of Simulink encapsulation structure can be called, the relationship between the three as shown in figure 4 :

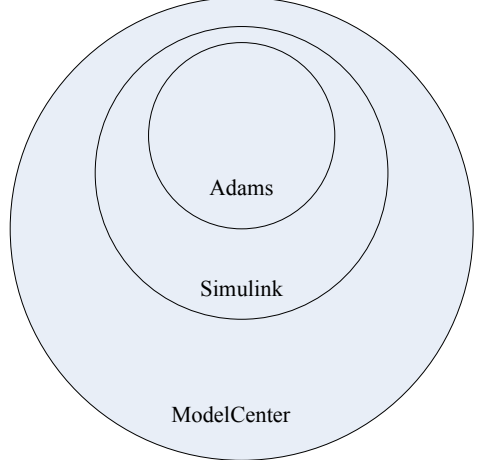

Figure 4 Adams, Simulink, ModelCenter and the relationship between the three

The Matlab module is added in the Modelcenter after, need to make the following processing:

(1)in the ModelCenter, because it contains a plug-in interface Matlab, so you can directly support Matlab *.m file transfer or code editor, but does not directly support Simulink *mdl file, need to use the command line *.mdl file in the system, add the Matlab plug-in, add the following key code:

Run dw3

Sim ('dw3 pid.mdl', 5)

Among them, dw3 is formed by Adams control module export *.m file, which contains the initialization information control module, the information must be loaded in advance to control the operation of the system; dw2 pid.mdl is in the control block diagram of the Simulink is established, followed by the number 5 for the simulation time setting.

(2)according to the military standard provisions, draw the conclusion that is composed of the following formula to calculate the stable precision of gun control system:

$\alpha=\sqrt{\frac{\sum_{i}^{n}\left(\bar{\alpha}-\alpha_{i}\right)^{2}}{n-1}}$

$\bar{\alpha}=\frac{\sum_{i=1}^{n}\left|\alpha_{i}\right|}{n}$ 
To the above formula is programmed, the need to add the following code in the Matlab module in ModelCenter:

Out=angle $(:, 2)$

Len=length (out)

$\mathrm{Dw}=0$

For $\mathrm{i}=1$ :len

$\mathrm{Dw}=\mathrm{dw}+$ out $(\mathrm{I})$;

End

Average $=\mathrm{dw} / \mathrm{len}$

Dw2 $=0$

For $\mathrm{j}=1$ :len

Dw2 $=$ dw2 $+($ out $(\mathrm{I})$-average $) *($ out $(\mathrm{I})$-average $)$;

End

Dw2 $=\mathrm{dw} 2 /($ len-1)

Result $=0.6745 *$ sqrt $(\mathrm{dw} 2)$

Among them, the array angle[] is barrel trunnion angle deviation relative to, from the angular velocity deviation integral to; result is calculated from the stable precision.

(3)in order to sensitivity analysis and optimization design for the next step, the construction of the variables Gain_P, Gain_I in ModelCenter, and add the following code:

$\mathrm{P}=$ Gain_P

$\mathrm{I}=$ Gain_I

Among them, $\mathrm{P}$ is the early establishment of control parameters of proportional coefficient in the block diagram, the I is the integral coefficient.

\section{SENSITIVITY ANALYSIS}

Select the PI controller parameters of proportion coefficient and integral coefficient is reasonable or not, directly related to the control effect, but also have a great impact on the level of machine torque demand, it is necessary to conduct sensitivity analysis on its influence, compare to the stabilization precision.

Sensitivity is the sensitivity of objective function to the design variables. Through the sensitivity analysis, can accurately evaluate the effects of various parameters on the system performance. Sensitivity analysis method is generally one order difference method, the adjoint variable method, direct differential method, this paper uses the method of first order difference of the sensitivity calculation model:

$\frac{\Delta f}{\Delta x}=\frac{f(x+\Delta x)-f(x)}{\Delta x}$

Variable selection for the proportion coefficient and integral coefficient controller, the dependent variable is the stable precision, sensitivity of the simulation results obtained as shown in figure 5,6:

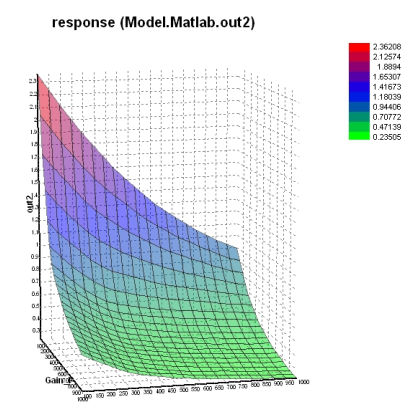

Figure 5 stable precision 3D response surface 


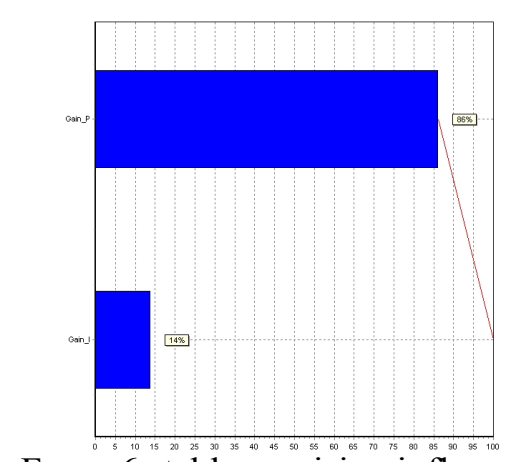

Fgure 6 stable precision influence

From an overall point of view, we can see from Figure 5, basically stable precision and two independent variables is inversely proportional relationship, i.e., with the increase in the proportion coefficient and integral coefficient, stable precision becomes smaller, the better the control effect.

From the individual point of view, we can see from Figure 6, the two independent variables are distinct influence on stability of accuracy, compared with the change of integral coefficient, variation coefficient of proportionality influence on steady precision obviously violently, therefore, in the structural design of the controller, to strictly control the proportion coefficients.

However, if it does not consider the actual factors (such as motor rated torque), and only the pursuit of the minimum stable accuracy goals, only two parameters can be increased indefinitely. But in fact, increasing the coefficient of the two controllers, and at the same time will increase the output torque, and the output torque and can not blindly increase, directly will be used by the rated motor torque limit. The control parameters of the system it is necessary to find an optimal configuration, which makes the gun control system in the premise of satisfying the torque constraints, to achieve the minimum stable precision.

\section{OPTIMIZATION DESIGN}

Internal ModelCenter provides a variety of optimization tools, which, design exploration optimizer (Design Explorer) integrated gradient optimization technique (Gradient Optimization), agent model (Surrogate Models) and experimental design (DOE) technology, not only can the design space sampling analysis system of effective, but also in the process of optimization of intelligent the use of agent model of iterative calculation, greatly improving the efficiency of optimization; in addition, especially suitable for solving a global discrete or continuous design variables optimization problems defined. Therefore, this paper selects it as the optimization tool overhead weapon station gun control system parameters.

On the overhead weapon station gun control system optimization simulation model, the calculation process for a total of 52 iterations, time-consuming 7 minutes, the optimization results as shown in table 1:

\begin{tabular}{lcl} 
& \multicolumn{2}{c}{ Table1 Optimization results } \\
parameter & initial & results \\
Gain_P & 336.67 & 91.35 \\
Gain_I & 336.67 & 497.13 \\
torque & 58.59 & 16.22 \\
result & 0.69 & 1.56
\end{tabular}

As shown in the table, the optimized proportion coefficient Gain_P is the result of 91.35, optimization of integral coefficient Gain_I is the result of 497.13, a difference of one order of magnitude, which is also in line with strict control of proportion system the number of values in the conclusion of the sensitivity analysis. In addition, although the final accuracy stability optimization result is 
$1.56 \mathrm{mil}$, compared with the initial value of $0.69 \mathrm{mil}$ to be big, but this is considered the optimization results of rated torque of the motor, with the authenticity, reliability, more reference value to engineering practice.

\section{CONCLUSION}

This paper constructs the overhead weapon station stability combined simulation precision electromechanical model through Adams, Simulink and other CAE software, and based on the multidisciplinary design optimization platform ModelCenter sensitivity analysis and design optimization of the model. The results show that the simulation of multi disciplinary collaborative overhead weapon station stabilization accuracy optimization model of short design cycle, high reliability, strong commonality based on, has the certain theory reference value, for the next step of overhead weapon station moving between the firing stability optimization accuracy to provide technical basis.

\section{REFERENCES}

[1]. Chen, Pengfei. 2014. Parameter optimization of precision air stage system modeling based on ModelCenter. modular machine tool \& automatic machining technology: 26-29

[2]. Ma, Chunmao. 1992. Effects of antiaircraft gun structure parameters on the muzzle vibration analysis. Journal of China Ordnance weapons Sciences: 24-27

[3]. Mao Baoquan.2012. Remote weapon station structural parameters optimization. Journal of The Academy of Armored Forces Engineering: 43-46

[4]. Mao Baoquan. Gun automatic weapon optimization design. National Defence Industry Press

[5]. Zhu Dequan. The joint simulation system of mechanical electrical hydraulic integration optimization design method. University of Science \& Technology 